\title{
Efeito do óleo essencial de manjericão (Ocimum basilicum L.) sobre o carrapato bovino Rhipicephalus (Boophilus) microplus em ensaios in vitro
}

\author{
Essential basil oil effect (Ocimum basilicum L.) on cattle tick \\ Rhipicephalus (Boophilus) microplus in in vitro experiments
}

\author{
Fernanda Carlini Cunha dos Santos ${ }^{1 *}$; Fernanda Silveira Flores Vogel ${ }^{2}$; \\ Silvia Gonzalez Monteiro ${ }^{3}$
}

\section{Resumo}

O uso excessivo de acaricidas predispõe a contaminação do meio ambiente, do homem e estimula a seleção de cepas de carrapatos resistentes. A maioria dos acaricidas químicos disponíveis no mercado apresenta eficácia reduzida devido ao intenso uso e manejo inadequado dos tratamentos. Sendo assim, alternativas ecologicamente viáveis utilizadas no controle dos carrapatos é uma área de pesquisa promissora. A fitoterapia tem como fundamento o estudo de plantas medicinais, assim como sua utilização no controle e tratamento de enfermidades tanto na área humana quanto na animal. Neste experimento foi avaliado o efeito in vitro do óleo essencial de manjericão (Ocimum basilicum) sobre fêmeas ingurgitadas de Rhipicephalus (Boophilus) microplus. Para isso foram testadas 6 concentrações do óleo de manjericão $(1 ; 5 ; 10 ; 25,50 ; 100 \%)$, diluído em água destilada, seguindo a metodologia do exame de biocarrapaticidograma. Perante os resultados foi observada inibição de postura de $10 ; 26 ; 26$; $33 ; 100$ e $100 \%$, eclosão larval de $75 ; 60 ; 80 ; 45 ; 0$ e $0 \%$ e a eficiência do tratamento de $28 ; 38 ; 36 ; 62$; 100 e $100 \%$, respectivamente. A avaliação estatística foi realizada pela análise de regressão linear, onde foi constatada associação significativa entre a concentração do óleo e a inibição de postura, eclosão larval e eficiência do tratamento. O óleo de manjericão á $25 \%$ obteve acima de $50 \%$ de eficiência, e nas concentrações de 50 e $100 \%$ obteve total inibição de postura com consequente eficiência máxima. Conforme os resultados, pode-se concluir que óleo de manjericão apresentou efeito acaricida in vitro sobre as teleóginas de $R$. (B) microplus, obtendo um controle parcial do parasito.

Palavras-chave: Carrapaticidas, fitoterapia, óleo essencial, produtos naturais, resistência parasitária

\begin{abstract}
The excessive use of acaricidal products induces environmental and human contamination, whereas there is also the selection of resistant tick's strains. Most of the chemical acaricides available in the market have low efficacy due intensive use and inadequate management of treatments. Thus, ecologically viable alternatives for the tick's control is a promising area of research. Herbal medicine has as fundamental issue the study of medicinal plants and their use in the control and treatment of human and animal diseases. This experiment has evaluated the in vitro efficacy of Ocimum basilicum essential oil on engorded teleogines of Rhipicephalus (Boophilus) microplus. Six concentrations of the
\end{abstract}

\footnotetext{
${ }^{1}$ Médica Veterinária Autônoma. Universidade Federal de Santa Maria, UFSM, Santa Maria, RS. E-mail: carlini@portoweb.com.br

${ }^{2}$ Prof $^{a}$ Dr $^{\mathrm{a}}$ do Dept $^{\circ}$ de Medicina Veterinária Preventiva, Laboratório de Doenças Parasitárias da UFSM, Santa Maria, RS. E-mail: fefevogel@gmail.com

${ }^{3}$ Prof $^{\mathrm{a}} \mathrm{Dr}^{\mathrm{a}}$ do Dept $^{\mathrm{o}}$ de Microbiologia e Parasitologia, Laboratório de Parasitologia Veterinária da UFSM, Santa Maria, RS. E-mail: sgmonteiro@uol.com

* Autor para correspondência
} 
oil $(1 ; 5 ; 10 ; 25,50 ; 100 \%)$ were tested, diluted in distilled water, using the engorged female immersion test. The inhibition of oviposition were $10 ; 26 ; 26 ; 33 ; 100$ and $100 \%$, the egg hatch were $75 ; 60 ; 80 ; 45$; 0 and $0 \%$, and the effectiveness of treatment $28 ; 38 ; 36 ; 62 ; 100$ and $100 \%$, respectively. The statistic evaluation was done by the linear regression test, and it was observed a significant association between the concentration of the oil and the inhibition of oviposition, egg hatch and effectiveness. The oil at $25 \%$ had higher than $50 \%$ of effectiveness, and at 50 and $100 \%$ had total inhibition of oviposition with maximum efficiency. According to the results, it is possible to conclude that the Ocimum basilicum oil had in vitro acaricidal action on $R$. (B) microplus teleogines, obtaining partial control of this parasite.

Key words: Essential oil, natural plants, parasitic resistance, phytoterapic

No Brasil, a principal espécie de carrapato que compromete a produtividade da pecuária bovina é o Rhipicephalus (Boophilus) microplus (SILVA et al., 2007). Esse ectoparasita é hematófago e seu principal hospedeiro é o bovino, podendo parasitar também ovinos, equinos e até mesmo seres humanos. Este ácaro encontra-se amplamente distribuído nos grandes rebanhos bovinos da América, África, Ásia e Oceania, entre os paralelos $32^{\circ} \mathrm{N}$ e $32^{\circ} \mathrm{S}$ (JOHNSTON; KEMP; PEARSON, 1986).

Santos Júnior, Furlong e Daemon (2000) relataram que não existe uma preocupação por parte dos proprietários no que concerne a eficiência dos tratamentos acaricidas. Este fato acarreta em fracassos no controle deste parasito e um aumento de custos, representado pelo elevado número de tratamentos. O uso excessivo de acaricidas predispõe a contaminação do meio ambiente, do homem e desenvolvimento de cepas de carrapatos resistentes. Devido às inúmeras dificuldades no controle e combate desses artrópodes, a utilização de plantas medicinais é uma alternativa cada vez mais viável em países em desenvolvimento como o Brasil, tornando-se cada vez mais importante o estudo para se descobrir plantas que possuam real efeito acaricida (SOARES, 2003). Algumas espécies vegetais são cultivadas desde a antiguidade visando à cura de doenças, podendo-se afirmar que o hábito de recorrer às propriedades de plantas medicinais é uma das primeiras manifestações do homem para compreender e utilizar a natureza (TESKE; TRENTINI, 1995). O manjericão (Ocimum basilicum L.) é uma planta perene, pertencente à família Lamiaceae, utilizada principalmente como tempero, planta ornamental e em chás. Existem alguns trabalhos que relatam o uso de óleo de manjericão como repelente (PADILHA et al., 2004), na ação na inibição do desenvolvimento e esporulação de fungos fitopatogênicos (SCHWANESTRADA; STANGARLI; CRUZ, 2000), no desenvolvimento micelial de fungos (PEREIRA et al., 2006) e na medicina popular como antiespasmódico, anti-inflamatório e calmante (VENDRUSCOLO; MENTZ, 2006). Esta planta possui inúmeros compostos químicos como linalol, estragol, farnesene, eugenol e cineol, sendo que todos estes são possuidores de propriedades repelentes (MARTINEZ-VELAZQUEZ et al., 2011). Diante deste contexto, o objetivo deste experimento foi avaliar a eficácia in vitro de diferentes concentrações de óleo essencial de manjericão (Ocimum basilicum) sobre teleóginas de $R$. (B) microplus.

Os testes in vitro foram conduzidos no Laboratório de Doenças Parasitárias da Universidade Federal de Santa Maria, Rio Grande do Sul (RS), entre os meses de junho a setembro de 2010. Foram coletadas 210 teleóginas de $R$. (B) microplus em bovinos naturalmente infestados em uma propriedade de Santa Maria, RS. No laboratório, as mesmas foram pesadas, lavadas em água corrente, secas em papel toalha e separadas em 10 grupos contendo 10 teleóginas de peso semelhante, seguindo a técnica de biocarrapaticidograma descrita por Drummond et al. (1973). O óleo essencial de manjericão foi adquirido da empresa Tecpon de Cachoerinha, RS, sendo que este é extraído da planta através do método de arraste de vapor. 
O experimento constituiu-se de 7 grupos, sendo o grupo I o controle, não tratado, e os grupos II ao VII correspondem às concentrações de $1 ; 5 ; 10$; 25; 50 e $100 \%$ do óleo essencial de manjericão, respectivamente, diluído em água destilada. O grupo controle foi imerso em água destilada seguindo a mesma metodologia utilizada nos tratamentos de II a VII. Para cada tratamento foram utilizadas 10 teleóginas, as quais foram imersas em $20 \mathrm{~mL}$ das soluções correspondentes a cada tratamento por 5 minutos. Após a imersão, as teleóginas foram secas em papel toalha e fixadas através de fita adesiva em placas de Petri, previamente identificadas. A seguir foram levadas a estufa climatizada, regulada a temperatura de $27{ }^{\circ} \mathrm{C}$, umidade relativa superior a $80 \%$, por 14 dias. Após o período de ovipostura, o total da postura foi pesada e $0,3 \mathrm{~g}$ desse conteúdo foi armazenado em tubos de ensaio, vedados com algodão hidrófilo, sendo, novamente, encaminhados para a estufa, onde foram mantidos por 26 dias. Após o período de incubação dos ovos, realizou-se a leitura do percentual de eclodibilidade. A eficácia do tratamento foi determinada a partir do cálculo do índice de inibição da reprodução, segundo Drummond et al. (1973). Todos os tratamentos foram realizados em triplicata e os resultados foram obtidos através de suas médias. A avaliação estatística foi realizada através do método de análise de regressão, a um nível de significância de 5\%.

A inibição de postura foi de $10 ; 26 ; 26 ; 33 ; 100$ e $100 \%$, respectivamente. A eclosão larval foi de 75 ; $60 ; 80 ; 45 ; 0$ e $0 \%$, respectivamente. E a eficiência do tratamento foi de $28 ; 38 ; 36 ; 62 ; 100$ e $100 \%$ respectivamente (Tabela 1). O óleo de manjericão em baixa concentração (1\%) afetou negativamente a oviposição e a eclosão larval. A concentração do óleo foi inversamente proporcional á postura e a eficiência do tratamento aumentou gradativamente, atingindo seu ponto máximo na concentração de $50 \%$ do óleo. A eclosão larval reduziu-se conforme o aumento da concentração, a exceção do tratamento com $10 \%$ do óleo. Isto pode ser atribuído ao efeito residual do produto, não afetando diretamente a postura dos ovos, mas alterando sua capacidade de eclosão e nova geração de larvas. O óleo á $25 \%$ obteve acima de $50 \%$ de eficiência de tratamento, apresentando controle parcial do carrapato bovino. As concentrações de 50 e $100 \%$ apresentaram total inibição de postura com consequente eficiência máxima.

Tabela 1. Médias percentuais (\%) de inibição de postura (IP), eclosão larval (EL), e eficiência do tratamento (ET) das diferentes concentrações do óleo de Ocimum basilicum ([ ]) em teleóginas de Rhipicephalus (Boophilus) microplus, Santa Maria, RS.

\begin{tabular}{ccccc}
\hline TRATAMENTO & {[]} & IP & EP & ET \\
\hline I & 0 & 0 & 100 & 0 \\
II & 1 & 10 & 75 & 28 \\
III & 5 & 26 & 60 & 38 \\
IV & 10 & 26 & 80 & 36 \\
V & 25 & 33 & 45 & 62 \\
VI & 50 & 100 & 0 & 100 \\
VII & 100 & 100 & 0 & 100 \\
\hline
\end{tabular}

Fonte: Elaboração dos autores.

Na análise de regressão linear com os dados de inibição de postura (x) e concentração de óleo de manjericão (y) foi verificada associação significativa $(\mathrm{P}<0,05)$ expressa na equação $\mathrm{y}=19,3+0,948 \mathrm{x}$
(Gráfico 1). Com os dados de eclosão de postura (x) e concentração do óleo (y) foi verificada associação significativa $(\mathrm{P}<0,05)$ expressa na equação y $=69,9$ - 0,833x (Gráfico 2). Com os dados de eficácia do 
tratamento (x) e concentração de óleo foi verificada reduzia e consequentemente a eficiência do associação significativa $(\mathrm{P}<0,05)$ expressa na tratamento também aumentava, sugerindo uma ação equação y $=36,6+0,766 x$. (Gráfico 3 ). Conforme do manjericão sobre a mortalidade ou reprodução a concentração do óleo de manjericão aumentava das teleóginas.

a inibição de postura aumentava, a eclosão larval

Gráfico 1. Análise de regressão linear com os parâmetros de inibição de postura (IP) (x) e concentração de óleo de Ocimum basilicum (\%) (y) em teleóginas de Rhipicephalus (Boophilus) microplus.

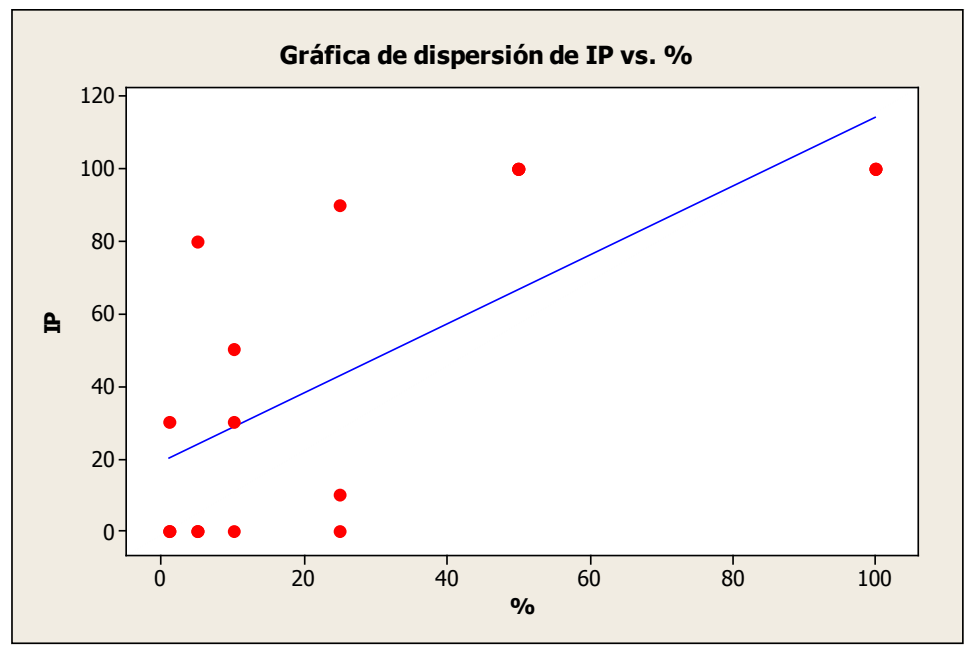

Fonte: Elaboração dos autores.

Gráfico 2. Análise de regressão linear com os parâmetros de eclosão da postura (EP) (x) e concentração de óleo de Ocimum basilicum (\%) (y) em teleóginas de Rhipicephalus (Boophilus) microplus.

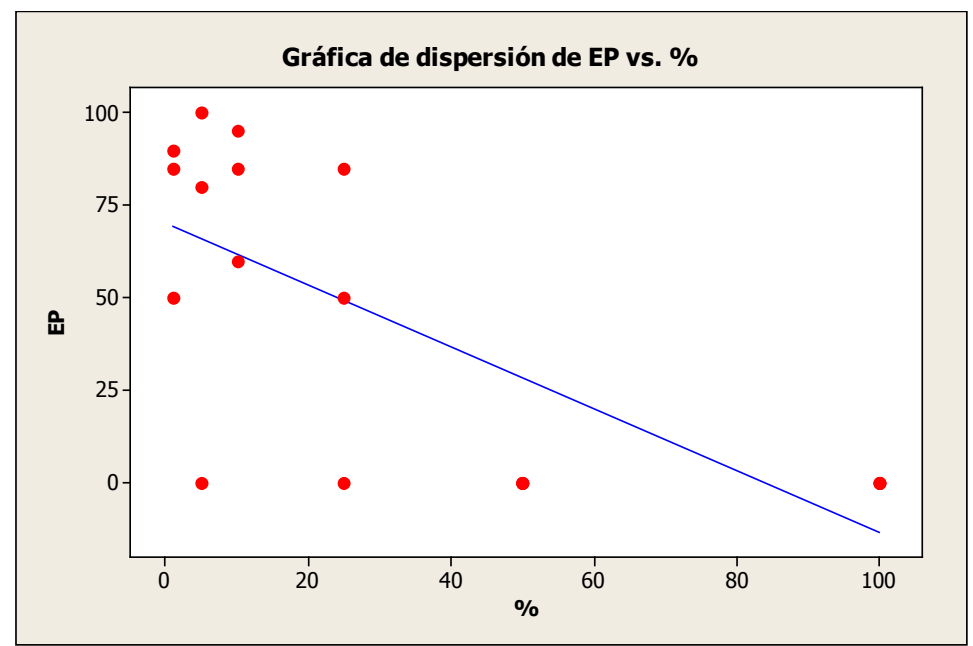

Fonte: Elaboração dos autores. 
Gráfico 3. Análise de regressão linear com os parâmetros de eficiência do tratamento (ET) (x) e concentração de óleo de Ocimum basilicum (\%) (y) em teleóginas de Rhipicephalus (Boophilus) microplus.

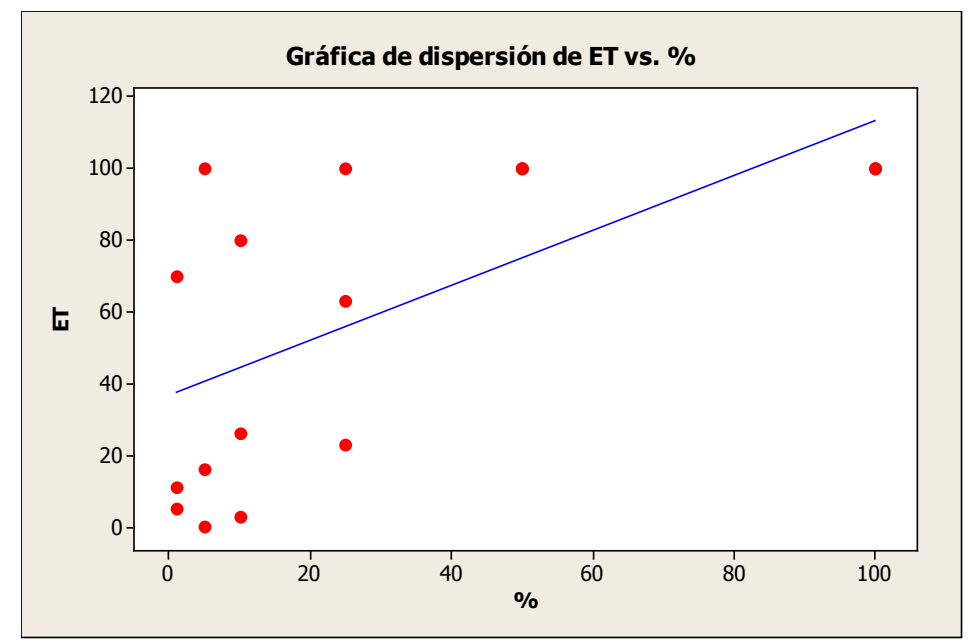

Fonte: Elaboração dos autores.

A ação repelente do $O$. basilicum também foi reportada por diferentes autores nas espécies Nephotettix virescens (CHITTIHUNSA; SAMNGAMIN, 1999), Anopheles stephensi, Aedes aegypti e Culex quinquefasciatus (PRAJAPATI et al., 2005) e inibição da alimentação em Helicoverpa armigera (RAO; PRATIBH; KORWAR, 2000). Diversos autores avaliaram óleos essenciais e compostos isolados de plantas do gênero Ocimum e constataram que estes fitoterápicos possuem ação inseticida e acaricida (MWANGI et al., 1995; MURUGAN; MURUGAN; NOORTHEN, 2007, KOSTIC et al., 2008; DEL FABRO; NAZZI, 2008).

Kostic et al. (2008) testando óleo essencial de manjericão sobre larvas de Lymantria dispar constataram que este fitoterápico apresentou baixa toxicidade e alta ação na inibição da alimentação das mariposas, provocando assim a sua morte. Mwangi et al. (1995) avaliaram o efeito do óleo essencial, obtido de folhas de $O$. suave, sobre larvas e adultos do carrapato Rhipicephalus appendiculatus e constataram que o óleo possui ação repelente e é tóxico para esta espécie. Del Fabro e Nazzi (2008) trabalharam com esta mesma planta apontaram para uma ação repelente sobre o carrapato Idoxes ricinus. Estes mesmo autores também avaliaram o efeito de compostos constituídos por eugenol e linaol e constataram alta ação repelente do eugenol e baixa do linalol.

Diversos estudos com diferentes extratos vegetais, avaliando a ação acaricida sobre $R$. (B) microplus, têm sido realizados no Brasil na tentativa de encontrar uma alternativa ecológica para o controle do carrapato.

Analisando diversos fitoterápicos, Costa et al. (2008) demonstraram que o extrato de Eucalyptus mostrou-se eficaz em testes in vitro sobre teleóginas de $R$. (B) microplus, apontando uma nova fonte de pesquisa com este fitoterápico. Avaliações feitas com óleo Cymbopogon winterianus (citronela de Java) demonstraram uma eficiência de $50 \%$ no controle de teleóginas e larvas, usando concentrações de 6,1 e $4,1 \%$. Foi verificado que não houve postura quando as teleóginas foram tratadas com o óleo a $10 \%$ e não houve eclosão larval na concentração de 7,14\% (MARTINS, 2006). No presente estudo, os resultados foram diferentes, uma vez que houve 60 e $80 \%$ de eclosão das larvas nas concentrações de 5 e $10 \%$, respectivamente. Isto indica que ambas plantas possuem ação sobre os carrapatos, no entanto o óleo de citronela de Java apresenta ação 
acaricida superior ao óleo de manjericão, mesmo em menor concentração.

Martinez-Velazquez et al. (2011) avaliaram o efeito de Cuminum cyminum, Pimenta dioica e $O$. basilicum sobre larvas de $R$. (B) microplus e constataram que as duas primeiras são tóxicas para esta espécie e que o óleo essencial de manjericão não apresentou efeito sobre as larvas. Os resultados do presente experimento discordam desta afirmação. As maiores eficiências foram obtidas através de alterações na inibição de postura e na eclosão larval, ou seja, devido á alterações na reprodução das teleóginas, porém sem causar a morte das mesmas. Esta constatação explicaria o porquê da divergência de resultados entre os autores, além da diferença entre o estágio de vida em que foram realizados os experimentos.

Soares (2003) testando Azadirachta indica (neem) in vitro sobre fêmeas ingurgitadas de $R$. (B) microplus obteve eficácia acima de 95\% tanto para solução aquosa quanto para alcoólica. No entanto, testando o Cymbopogon citratus (capim cidreira) obteve eficácia de apenas 48\%. Segundo estes dados, pode-se sugerir que a divergência nos resultados obtidos pelo autor podem ser atribuídos ao diferente potencial acaricida de cada planta, ao meio de ação de seus princípios ativos e a forma de extração de seu óleo. Mais recentemente, Olivo et al. (2009) verificaram que formulações contendo extrato aquoso de Nicotiana tabacum obtido por decocção, apresentaram um controle parcial do carrapato em bovinos naturalmente infestados. Assim como no presente experimento, existem diversos fitoterápicos que em baixas concentrações que não apresentam $100 \%$ de eficácia sobre o $R$. (B) microplus, mas poderiam ser utilizados de forma auxiliar no seu controle, uma vez que possuem ação acaricida e apresentam controle parcial do parasita.

O uso de fitoterápicos é uma interessante opção para diminuir a aplicação de acaricidas químicos, embora geralmente apresentem uma eficácia inferior a estes produtos. No entanto, novos estudos com o óleo essencial de Ocimum basilicum devem ser conduzidos a fim de avaliar sua resposta in vivo, toxicidade, identificação dos compostos com maior atividade acaricida e a possibilidade de conseguir bons resultados com concentrações mínimas. Os resultados indicam a existência de ação negativa do óleo essencial de Ocimum basilicum em relação a parâmetros reprodutivos de teleóginas de Rhipicephalus (Boophilus) microplus in vitro e apresentando controle parcial do parasita.

\section{Referências}

CHITTIHUNSA, T.; SAMNGAMNIM, N. Studies on the effects of some crude plant extracts to the mortality and feeding behavior of Nephotettix virescens. In: OATES, C. G. (Ed.). KASETSART UNIVERSITY ANNUAL CONFERENCE, 37., Bangkok, Thaliand, Proceedings... Bangkok: Text and Journal Publication, 1999. p. 70-77.

COSTA, F. B.; VASCONCELOS, P. S. da S.; SILVA, A. M. M.; BRANDÃO, V. M.; SILVA, I. A. da; TEIXEIRA, W. C.; GUERRA, R. M. S. N.; DOS SANTOS, A. C. G. Eficácia de fitoterápicos em fêmeas ingurgitadas de Boophilus microplus, provenientes da mesorregião Oeste do Maranhão, Brasil. Revista Brasileira de Parasitologia Veterinária, Jaboticabal, v. 17, n. 1, p. 83-86, 2008.

DEL FABRO, S.; NAZZI, F. Repellent effect of sweet basil compounds on Idoxes ricinus ticks. Experimental and Applied Acarology, Amsterdam, v. 45, n. 3, p. 219228, 2008.

DRUMMOND, R. O.; ERNST, S. E.; TREVINO, J. L.; GLADNEY, W. J.; GRAHAM, O. H. Boophilus annulatus and Boophilus microplus: laboratory tests for insecticides. Journal of Economic Entomology, Lanham, v. 66, n. 1, p. 130-133, 1973.

JOHNSTON, L. A. Y.; KEMP, D. H.; PEARSON, R. D. Immunization of cattle against Boophilus microplus using extracts derived from adult female ticks: effects of induced immunity on tick populations. International Journal for Parasitology, New York, v. 16, n. 1, p. 27-34, 1986.

KOSTIC, M.; POPOVIC, Z.; BRKIC, D.; MILANOVIC, S.; SIVCEV, I.; STANKOVIC, S. Larvicidal and antifeedant activity of some plant-derived compounds to Lymantria dispar L. (Lepidoptera: Limantriidae). Bioresource Technology, Miramar, v. 99, n. 16, p. 78977901, 2008. 
MARTINEZ-VELAZQUEZ， G. A.; CASTILLOHERRERA, R.; ROSARIO-CRUZ, J. M.; FLORESFERNANDEZ, J.; LOPEZ-RAMIREZ, R.; HERNANDEZ-GUTIERREZ, E.; LUGO-CERVANTES, C. Acaricidal effect and chemical composition of essential oils extracted from Cuminum cyminum, Pimenta dioica and Ocimum basilicum against the cattle tick Rhipicephalus (Boophilus) microplus (Acari: Ixodidae). Parasitology Research, Berlim, v. 108, n. 2, p. 481-487, 2011.

MARTINS, R. M. Estudo in vitro da ação acaricida do óleo essencial da gramínea Citronela de Java (Cymbopogon winterianus Jowitt) no carrapato Boophilus microplus. Revista Brasileira de Plantas Medicinais, Botucatu, v. 8, n. 2, p. 71-78, 2006.

MURUGAN, K.; MURUGAN, P.; NOORTHEN, A. Larvicidal and repellent potential of Albizzia amara Boivin and Ocimum basilicum Linn against dengue vector Aedes aegypti (Insecta: Diptera: Culicidae), Bioresource Technology, Miramar, v. 98, n. 1, p. 198-201, 2007.

MWANGI, E. N.; HASSANALI, A.; ESSUMAN, S.; MYANDAT, E.; MOREKA, L.; KIMONDO, M. Repellent and acaricidal properties of Ocimum suave against Rhipicephalus appendiculatus ticks. Experimental and Applied Acarology, Amsterdam, v. 19, n. 1, p. 11-18, 1995.

OLIVO, C. J.; HEIMENDINGER, A.; ZIECH, M. F.; AGNOLIN, C. A.; MEINERZ, G. R.; BOTH, F.; CHARÃO, P. S. Extrato aquoso de fumo em corda no controle do carrapato de bovinos. Ciência Rural, Santa Maria, v. 39, n. 4, p. 1131-1135, 2009.

PADILHA, F. P.; FARAGO, P. V.; CECCHIA, L. P. M.; HIROSE, K. M.; RIBAS, J. L. C. Estudo da ação repelente do óleo essencial de Ocium selloi Benth contra o Anopheles Brasiliensis. Acta Farmaceutica Bonaerense, Buenos Aires, v. 23, n. 3, p. 376-378, 2004.

PEREIRA, M. C.; VILELA, G. R.; COSTA, L. M. A. S.; SILVA, R. F.; FERNANDES, A. F.; FONSECA, E. W. N.; PICCOLI, R. H. Inibição do desenvolvimento fúngico através da utilização de óleos essenciais de condimentos. Ciência Agrotécnica, Lavras, v. 30, n. 4, p. 731-738, 2006.
PRAJAPATI, V.; TRIPATHI, A. K.; AGGARWAL, K. K.; KHANUJA, S. P. S. Insecticidal, repellent and ovipostion-derrent activity of selected essential oils against Anopheles stephensi, Aedes aegypti e Culex quinquefasciatus. Bioresource Technology, Miramar, v. 99, n. 16, p. 1897-1901, 2005.

RAO, M. S.; PRATIBH, G.; KORWAR, G. R. Evaluation of aromatic oils against Helicoverpa armigera. Annals of Plant Protection Science, New Jershey, v. 87, n. 8, p. 236-238, 2000.

SANTOS JÚNIOR, J. C.; FURLONG, J.; DAEMON, E. Controle do carrapato Boophilus microplus (acari:Ixodidae) em sistemas de produção de leite da microrregião fisiográfica Fluminense do grande Rio-Rio de Janeiro. Ciência Rural, Santa Maria, v. 30, n. 2, p. 305-311, 2000.

SCHWAN-ESTRADA, K. R. F.; STANGARLI, J. R.; CRUZ, M. E. R. Uso de extratos vegetais no controle de fungos fitopatogênicos. Floresta, Curitiba, v. 30, n. 1-2, p. $129-137,2000$.

SILVA, W. W.; ATHAYDE, A. C. R.; RODRIGUES, O. G.; ARAÚJO, G. M. B.; SANTOS, V. D.; NETO, A. B. S.;COELHO, M. C. O. C.; MARINHO, M. L. Efeitos do neem (Azadirachta indica A. Juss) e do capim santo [Cymbopogon citratus (DC) Stapf] sobre os parâmetros reprodutivos de fêmeas ingurgitadas de Boophilus microplus e Rhipicephalus sanguineus (Acari: Ixodidae) no semiárido paraibano. Revista Brasileira Plantas Medicinais, Botucatu, v. 9, n. 3, p. 1-5, 2007.

SOARES, M. C. S. C. Avaliação comparativa da eficácia de fitoterápicos e produtos químicos carrapaticidas no controle de Boophilus microplus (Canestrini, 1887) por meio do biocarrapaticidograma. 2003. Dissertação (Mestrado em Ciência Veterinária) - Departamento de Medicina Veterinária. Universidade Federal Rural de Pernambuco, Recife.

TESKE, M.; TRENTINI, A. M. Herbarium compêndio de fitoterapia. 2. ed. Curitiba: Herbarium Laboratório Botânico, 1995. 317 p.

VENDRUSCOLO, G. S.; MENTZ, L. A. Estudo da concordância das citações de uso e importância das espécies e famílias utilizadas como medicinais pela comunidade do bairro. Acta Botânica Brasílica, Feira de Santana, v. 20, n. 2, p. 367-382, 2006. 
